\title{
Thyroid lobectomy in patients with differentiated thyroid cancer: an analysis of the clinical outcomes in a nationwide multicenter study
}

\author{
Juan J. Díez ${ }^{1,2}$, Victoria Alcázar ${ }^{3}$, Pedro Iglesias ${ }^{1,2}$, Ana Romero-Lluch ${ }^{4}$, Julia Sastre ${ }^{5}$, \\ Begoña Pérez Corral ${ }^{6}$, Carles Zafón ${ }^{7}$, Juan Carlos Galofré ${ }^{8}$, María José Pamplona ${ }^{9}$
}

${ }^{1}$ Department of Endocrinology, Hospital Universitario Ramón y Cajal, Madrid, Spain; ${ }^{2}$ Department of Endocrinology, Hospital Universitario Puerta de Hierro Majadahonda, Instituto de Investigación Sanitaria Puerta de Hierro Segovia de Arana, Majadahonda, Madrid, Spain; ${ }^{3}$ Department of Endocrinology, Hospital Universitario Severo Ochoa, Leganés, Madrid, Spain; ${ }^{4}$ Department of Endocrinology, Hospital Universitario Virgen del Rocío, Sevilla, Spain; ${ }^{5}$ Department of Endocrinology, Complejo Hospitalario de Toledo, Toledo, Spain; ${ }^{6}$ Department of Endocrinology, Complejo Asistencial Universitario de León, León, Spain; ${ }^{7}$ Department of Endocrinology, Hospital Universitari Vall d'Hebron, Barcelona, Spain; ${ }^{8}$ Department of Endocrinology, Clínica Universidad de Navarra, Pamplona, Spain; ${ }^{9}$ Department of Endocrinology, Hospital Royo Villanova, Zaragoza, Spain Contributions: (I) Conception and design: JJ Díez, P Iglesias; (II) Administrative support: All authors; (III) Provision of study materials or patients: All authors; (IV) Collection and assembly of data All authors; (V) Data analysis and interpretation: All authors; (VI) Manuscript writing: All authors; (VII) Final approval of manuscript: All authors.

Correspondence to: Juan J. Díez. Department of Endocrinology, Hospital Universitario Puerta de Hierro Majadahonda, Instituto de Investigación Sanitaria Puerta de Hierro Segovia de Arana, Calle Manuel de Falla, 1, 28222 Majadahonda, Madrid, Spain. Email: juanjose.diez@salud.madrid.org.

\begin{abstract}
Background: Total thyroidectomy is the standard initial surgery for differentiated thyroid carcinoma (DTC), but the extent of the thyroidectomy remains controversial. Thyroid lobectomy (TL) has been widely used in eastern countries; however, its use has not been generalized in western countries, including Spain. Our aims were to analyse the clinical outcome of a multicentre nation-wide cohort of DTC patients treated by TL and to assess the proportion of patients who required completion of the thyroidectomy and who presented disease recurrence.

Methods: We retrospectively analyzed patients who underwent TL for DTC and were followed-up for $\geq 12$ months. We collected demographic, clinical, and histopathological data. Dynamic risk stratification (DRS) was performed at 12 months and at last visit.

Results: One hundred and sixty-four patients (128 women, mean age 50.8 years, median follow-up 45.4 months) from 9 hospitals were included. There were 158 cases of papillary and 6 of follicular thyroid carcinoma (FTC). Remission of the disease (excellent response) was shown in $71.6 \%$ of the patients at 12 months and in $74.4 \%$ at the end of follow-up. At that time, there were 34 patients $(20.7 \%)$ with indeterminate response, $6(3.7 \%)$ with biochemical incomplete response, and $2(1.2 \%)$ with structural incomplete response. Completion of the thyroidectomy was necessary in 8 patients $(4.9 \%)$, but only 3 of them $(1.8 \%)$ had disease recurrence.

Conclusions: These results, obtained in real clinical practice, suggest that TL is a safe operative option for selected patients with DTC and that the intensity of the treatment must be tailored according to the presurgical tumor-associated risk, in line with a personalized medicine.
\end{abstract}

Keywords: Differentiated thyroid carcinoma (DTC); thyroid surgery; lobectomy; dynamic risk stratification (DRS); tumor recurrence

Submitted Sep 11, 2020. Accepted for publication Nov 04, 2020.

doi: $10.21037 /$ gs-20-712

View this article at: http://dx.doi.org/10.21037/gs-20-712

$\wedge$ ORCID: 0000-0002-2017-0694. 


\section{Introduction}

For many years, total thyroidectomy with or without radioiodine ablation has been the standard treatment for most patients with differentiated thyroid carcinoma (DTC). However, recently different studies have shown that less aggressive surgical interventions can be a valid alternative in low-risk patients, without impacting overall survival (1-4).

Diverse investigators consider that thyroid lobectomy (TL) is an adequate approach for a large proportion of patients with papillary (PTC) (5-8) and follicular thyroid carcinoma (FTC) $(9,10)$. The last version of the American Thyroid Association (ATA) guidelines (11) recognizes that the extent of initial thyroid surgery probably has little impact on disease-specific survival in properly selected lowto intermediate-risk patients and endorses TL as initial surgical therapy for DTCs up to $4 \mathrm{~cm}$ in the absence of lymph node involvement and extrathyroidal extension, although it recommends that this treatment decision be made by a multidisciplinary team. In countries such as Japan or Korea $(8,12,13)$ TL has been the mainstay of primary surgery for DTC, however, in western countries, including Spain, this procedure has not been widely used $(14,15)$.

In the present study, we set out to analyze the clinical outcomes of a nationwide multicenter cohort of patients with DTC, treated by TL, to assess the results of therapy using the modified dynamic risk stratification (DRS) criteria $(16,17)$, and to quantify the proportion of patients who required completion of thyroidectomy and who exhibited tumor recurrence. We present the following article in accordance with the STROBE reporting checklist (available at http://dx.doi.org/10.21037/gs-20-712).

\section{Methods}

\section{Patients}

We retrospectively studied patients who underwent TL for DTC. The following inclusion criteria were considered: surgical intervention performed in a surgical department of the participating hospital, available histological record, and duration of follow-up $\geq 12$ months after surgical treatment. Patients with any of the following criteria were excluded: age $<18$ years at the time of diagnosis, histology other than DTC, clinically evident local or distant metastasis at the time of initial diagnosis, absence of histological report, follow-up time $<12$ months, and absence of clinical and analytical data in the patient's medical record. Data collection was carried out in the participating hospitals between 2017 and 2019. The criterion for TL was based on the decision of the attending multidisciplinary team based on the ATA 2015 guidelines recommendations (11).

This study was conducted in accordance with the Declaration of Helsinki (as revised in 2013). The study was approved by the board of directors of the Thyroid Area of the Spanish Society of Endocrinology and Nutrition (TiroSEEN) and the ethics committee of the Hospital Universitario Ramón y Cajal (Madrid) (Acta 334, 27/11/17). After approval, all TiroSEEN members were invited to participate. Since this is a non-intervention and exclusively retrospective analysis of data from routine clinical practice, the informed consent of the patients has not been considered necessary.

\section{Study design}

A data collection document was designed that included the following information: demographic and clinical data (hospital, sex, age, family history of thyroid cancer and preoperative diagnosis); histopathological data (histology type, tumor size, multifocality, invasiveness, incidental finding, extrathyroidal extension, presence of chronic autoimmune thyroiditis in non-tumor thyroid); initial risk classification (TNM classification and ATA risk of recurrence); follow-up data (duration of follow-up, ultrasound data, serum thyroglobulin $(\mathrm{Tg})$ concentration, thyroglobulin antibody (TgAb) titer; DRS at 12 months and at the end of follow-up); and finally, the need to complete thyroidectomy and the presence or absence of thyroid cancer in the second surgery. The follow-up period was considered until the last visit date or, when appropriate, until the date of completion thyroidectomy.

\section{Classification of patients}

For the initial classification of patients, we used the TNM staging system according to the criteria of the American Joint Committee on Cancer (AJCC/UICC), 8th ed. (18). For the initial classification of the risk of recurrence, the ATA 2009 criteria were used, with the modifications proposed in 2015 (11).

To assess the response to therapy, the DRS was used both at 12 months and at the end of follow-up. Four types of response were considered $(16,17,19)$ : excellent (stable $\mathrm{Tg}$ level $<30 \mathrm{ng} / \mathrm{mL}$ and negative $\mathrm{TgAb}$ and negative imaging), biochemical incomplete $(\mathrm{Tg}>30 \mathrm{ng} / \mathrm{mL}$ or increasing $\mathrm{Tg}$ levels or increasing TgAb levels and negative imaging), 
structural incomplete (evidence of disease regardless of $\mathrm{Tg}$ or $\mathrm{TgAb}$ ), and indeterminate response (nonspecific findings on imaging studies or TgAb levels stable or declining in the absence of structural disease). For the comparison of patients, two groups were considered: in remission (excellent response), and not cured (any other type of response).

\section{Laboratory and imaging tests}

In this retrospective study, data from the analytical and imaging tests performed in the usual clinical practice of the participating hospitals were collected. Although the protocols of each center were not identical, in most patients, laboratory (serum $\mathrm{Tg}$ and $\mathrm{TgAb}$ ) and imaging tests (neck ultrasound) were performed every 3-6 months for the first year and then annually or every 2 years. Serum $\mathrm{Tg}$ and $\mathrm{TgAb}$ levels were quantified according to the standard methods used in the clinical laboratories of each participating hospital. The most commonly used equipments for $\mathrm{Tg}$ measurement were Immulite (Siemens), Cobas (Roche) and Access (Beckman), with functional sensitivities between 0.1 and $0.9 \mathrm{ng} / \mathrm{mL}$. For the quantification of $\mathrm{TgAb}$, the most used appliances were Immulite and Advia Centaur (Siemens), Cobas (Roche), Access (Beckman) and Architect (Abbott). The cutoffs for TgAb positivity were different in the several employed assays, ranging between 4 and $60 \mathrm{mU} / \mathrm{L}$ in the participating hospitals. Given the diversity of methods, only the presence of positive or negative $\mathrm{TgAb}$ values was considered. In patients with positive $\mathrm{TgAb}$, the serum $\mathrm{Tg}$ value was not considered.

For ultrasound assessment during follow-up, the presence of nodules was considered when they were $>5 \mathrm{~mm}$. Nodular growth was defined as an increase of more than $20 \%$ in two dimensions. The following signs of suspicion were contemplated: microcalcifications, marked hypoechogenicity, irregular margins or absence of halo, shape taller than wide in cross sectional imaging, and central vascularization $(20,21)$. In the evaluation of the lymph nodes, the following signs of suspicion were taken into account: long/short axis index $<2.0$, focal or diffuse hyperechogenicity, micro- or macrocalcifications, cystic changes and loss of the fatty hilum (22).

\section{Statistical analysis}

Quantitative data are expressed as mean \pm SD for variables with normal distribution and as median (interquartile range) for non-parametric data. Adjustment to normal distribution was tested by the Kolmogorov-Smirnov test. Categorical variables are described as ratios or percentages. For comparison of means, the Student's $t$-test and Mann Whitney $U$ tests were used, as necessary. For ratio comparisons, the chi-square test or Fisher's exact test was used. Differences were considered significant when $\mathrm{P}<0.05$.

\section{Results}

\section{Studied patients}

Nine researchers from TiroSEEN agreed to participate in the study. Of the 199 patients contributed to the study, 35 were eliminated for the following reasons: duration of follow-up $<12$ months (19 patients), histology different from DTC (7 patients), absence of clinical data during follow-up (7 patients) and absence of histological report (2 patients).

One hundred and sixty-four patients (128 women) were included in the study (Table 1). Of the 158 cases with PTC, the majority presented the classic (97 cases) or follicular (39 cases) variants. The 6 patients with FTC were minimally invasive follicular thyroid cancers without angioinvasion. Age was significantly higher in men $(55.5 \pm 13.0 \mathrm{vs}$. $49.5 \pm$ 15.1 years, $\mathrm{P}<0.05)$. Duration of follow-up was shorter in men than in women, but without reaching statistical significance [34.8 (20.4-55.9) vs. 47.2 (24.1-70.7) months, $\mathrm{P}=0.132]$. All the other clinical and histopathological characteristics that appear in Table 1, were similar in both sexes.

\section{DRS}

The different types of response in the DRS and their changes between 12 months and the end of follow-up are depicted in Figure 1 and Table S1. In the 148 patients who underwent DRS at 12 months, excellent response was observed in $106(71.6 \%)$ and presence of disease (any other response) in 42 (28.4\%). Comparison of both groups of subjects showed that the latter were older $(54.9 \pm 14.3 \mathrm{vs}$. $49.5 \pm 4.4$ years, $\mathrm{P}<0.05)$, and had a higher proportion of chronic lymphocytic thyroiditis $(47.6 \%$ vs. $17.9 \%, \mathrm{P}<0.01)$ and nodules in the remaining thyroid lobe $(47.6 \%$ vs. $24.5 \%, \mathrm{P}<0.05)$. There were no differences in other clinical or histopathological characteristics (Table S2).

At last visit, 122 of 164 patients $(74.4 \%)$ were in remission and 42 (25.6\%) were not in remission. Patients with persistent disease exhibited a higher proportion 
Table 1 Clinical and histopathological characteristics of the studied patients

\begin{tabular}{|c|c|c|c|c|}
\hline Characteristics & All $(n=164)$ & Females $(n=128)$ & Males $(n=36)$ & $\mathrm{P}$ \\
\hline Duration of follow-up, months & $45.4(23.4-67.1)$ & $47.2(24.1-70.7)$ & $34.8(20.4-55.9)$ & 0.132 \\
\hline Family history of thyroid cancer & $8(5.0)$ & $6(4.7)$ & $2(5.6)$ & 1.000 \\
\hline Histological type & & & & 1.000 \\
\hline Follicular & $6(3.7)$ & $5(3.9)$ & $1(2.8)$ & \\
\hline Tumor size, $\mathrm{cm}$ & $0.4(0.2-0.7)$ & $0.40(0.25-0.70)$ & $0.33(0.20-0.79)$ & 0.638 \\
\hline Location & & & & 0.836 \\
\hline Right & $97(59.1)$ & $75(58.6)$ & $22(61.1)$ & \\
\hline Chronic lymphocytic thyroiditis & $42(25.6$ & $37(28.9)$ & $5(13.9)$ & 0.084 \\
\hline Multifocality & & & & 0.687 \\
\hline Unifocal & $156(95.1)$ & $121(94.5)$ & $35(97.2)$ & \\
\hline Multifocal & $8(4.9)$ & $7(5.5)$ & $1(2.8)$ & \\
\hline Incidental & $127(77.4)$ & $97(75.0)$ & $30(83.3)$ & 0.378 \\
\hline Extrathyroidal extensión & $0(0)$ & $0(0)$ & $0(0)$ & 1.000 \\
\hline TNM & & & & 0.267 \\
\hline $\mathrm{T} 1 \mathrm{a}$ & $153(93.3)$ & $119(93.0)$ & $34(94.4)$ & \\
\hline Low & $159(97.0)$ & $124(96.6)$ & $35(97.2)$ & \\
\hline Intermediate & $5(3.0)$ & $4(3.1)$ & $1(2.8)$ & \\
\hline Nodules at remnant lobe ${ }^{a}$ & $52(31.7)$ & $41(32.0)$ & $11(30.6)$ & 1.000 \\
\hline Size of the largest nodule in remnant lobe, $\mathrm{cm}^{\mathrm{b}}$ & $1.0(1.6-1.3)$ & $1.0(0.6-1.3)$ & $0.9(0.8-1.3)$ & 0.343 \\
\hline
\end{tabular}

of chronic autoimmune thyroiditis demonstrated in histopathological study than patients in remission $(42.9 \%$ vs. $19.7 \%$ ), with no other significant differences in the clinical and histopathological data summarized in Table 2.

\section{Persistent disease at the end of follow-up}

At the end of follow-up, there were 34 patients with indeterminate response (31 PTC and 3 FTC) (Figure S1). The criteria for this response were the presence of TgAb (18 


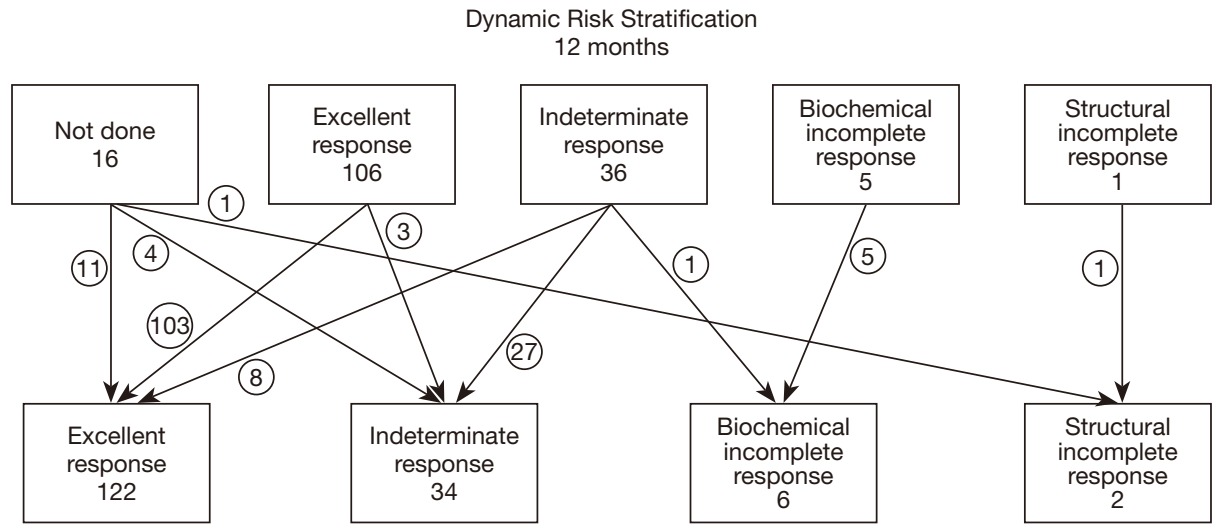

Last visit

Figure 1 Dynamic risk stratification performed 12 months after thyroid lobectomy (148 patients) and at the end of the follow-up period, indicating the changes in response between one time and another.

Table 2 Comparison of patients at the end of the follow-up period classified according to absence (excellent response) or presence of disease (any other type of response)

\begin{tabular}{|c|c|c|c|c|}
\hline Characteristics & No. & Excellent response $(n=122)$ & Any other response $(n=42)$ & $P$ \\
\hline Duration of follow-up, months & 164 & $46.4(24.2-67.6)$ & $40.9(22.1-73.4)$ & 0.862 \\
\hline Gender & 164 & & & 0.517 \\
\hline Female & & $97(79.5)$ & $31(73.8)$ & \\
\hline Histological type & 164 & & & 0.176 \\
\hline Papillary & & $119(97.5)$ & $39(92.9)$ & \\
\hline Follicular & & $3(2.5)$ & $3(7.1)$ & \\
\hline Tumor size, $\mathrm{cm}$ & 164 & $0.4(0.25-0.7)$ & $0.4(0.2-0.7)$ & 0.875 \\
\hline Unifocal & & $116(95.1)$ & $40(95.2)$ & \\
\hline Multifocal & & $6(4.9)$ & $2(4.8)$ & \\
\hline Incidental & 164 & $90(73.8)$ & $37(88.1)$ & 0.058 \\
\hline Central lymph node dissection & 163 & $3(2.5)$ & $2(4.8)$ & 0.604 \\
\hline Risk of recurrence ATA 2015 & 164 & & & 0.330 \\
\hline Low & & $117(95.9)$ & $42(100.0)$ & \\
\hline Intermediate & & $5(4.1)$ & $0(0)$ & \\
\hline Nodules at remnant lobe & 163 & $34(28.1)$ & $18(42.9)$ & 0.086 \\
\hline
\end{tabular}

Data are the mean \pm SD or the median (interquartile range) for quantitative variables, and the number (percentage) for categorical variables. ATA, American Thyroid Association. 
Table 3 Main characteristics of patients with biochemical incomplete and structural incomplete response at the end of the follow-up period

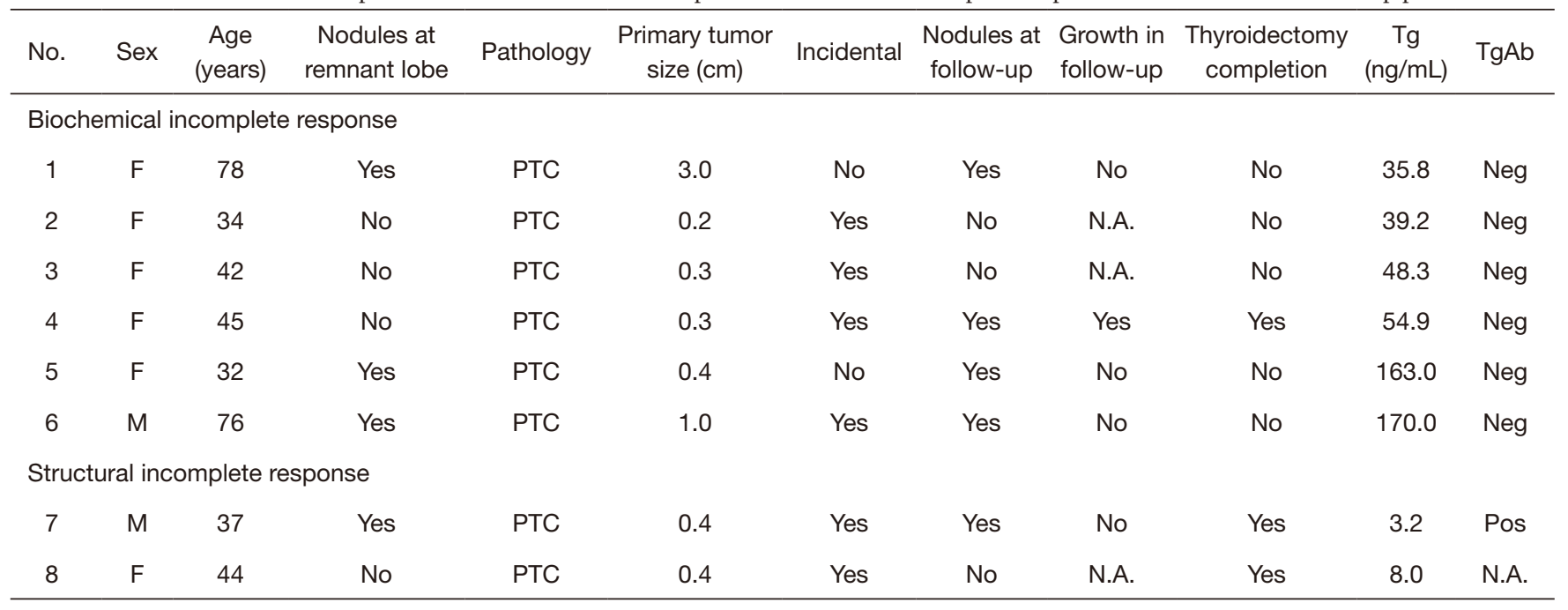

No., patient number; F, female; M, male; PTC, papillary thyroid carcinoma; Tg, thyroglobulin; TgAb, thyroglobulin antibodies; N.A., not available; Neg, negative; Pos, positive.

patients with PTC), ultrasound criteria (11 patients, 9 PTC and 2 FTC) and both criteria (5 patients, 4 PTC and 1 FTC). There were 6 patients with biochemical incomplete response (5 women, ages 32-78 years), all of them with PTC (size $0.2-3.0 \mathrm{~cm}$ ) and Tg concentrations between 35.8 and $170.0 \mathrm{ng} / \mathrm{mL}$. Four of these patients had nodules in the remnant lobe during follow-up, but only 1 showed growth. Thyroidectomy was completed in only one patient (Table 3). Of the 2 patients with structural incomplete response (Table 3), one had nodules during follow-up without growing. Thyroidectomy was completed in both.

\section{Ultrasound examinations}

During follow-up, ultrasound tests showed nodules in the remnant contralateral thyroid lobe in 60 patients $(36.6 \%)$ and absence of nodules in $102(62.2 \%)$. This information was not available in 2 patients $(1.2 \%)$. Of the 60 patients with nodules only 18 experienced growth. Of these, the thyroidectomy was completed in 3 patients without the finding of cancer in the specimen. Four thyroidectomy completions were performed in the 42 patients without nodular growth. Of these, tumor recurrence was observed in 2 cases. A further thyroidectomy was performed in one subject from the group of 102 patients without nodules during follow-up, also resulting in tumor recurrence (Figure S2). Table 4 shows the comparison of patients with and without nodular growth. We did not find any significant difference between both groups.

\section{Thyroidectomy completion}

Table 5 summarizes the characteristics of the 8 patients $(4.9 \%)$ in whom the thyroidectomy was completed (5 women, ages 20-67 years). All cases were incidental PTCs with sizes $0.2-2.0 \mathrm{~cm}$. Seven exhibited nodules during follow-up and growth was observed in 3. The reasons for completing thyroidectomy were suspicious nodules in 4 patients, suspicious lymph nodes in one patient, nodular growth in one patient, and elevated $\mathrm{Tg}$ in one patient. In one patient with excellent response, thyroidectomy was completed due to the diagnosis of primary hyperparathyroidism with surgical criteria. Fine needle aspiration cytology was available in 5 patients before thyroidectomy completion. Results confirmed malignancy in two of these patients.

Only 3 patients $(1.8 \%)$ showed thyroid cancer in the pathological specimen. The site of recurrence was the residual contralateral lobe in all 3 cases. One of them also had a recurrence in the central compartment. None had recurrence in the bed of the previously excised lobe or in the lateral compartment. No patient died during the followup period.

\section{Discussion}

Results of the present multicenter study show a favorable evolution of patients with DTC treated with TL. Most of them (71.6\%) achieve an excellent response one year 
Table 4 Comparison of 60 patients with thyroid nodules during follow-up classified according to growth pattern

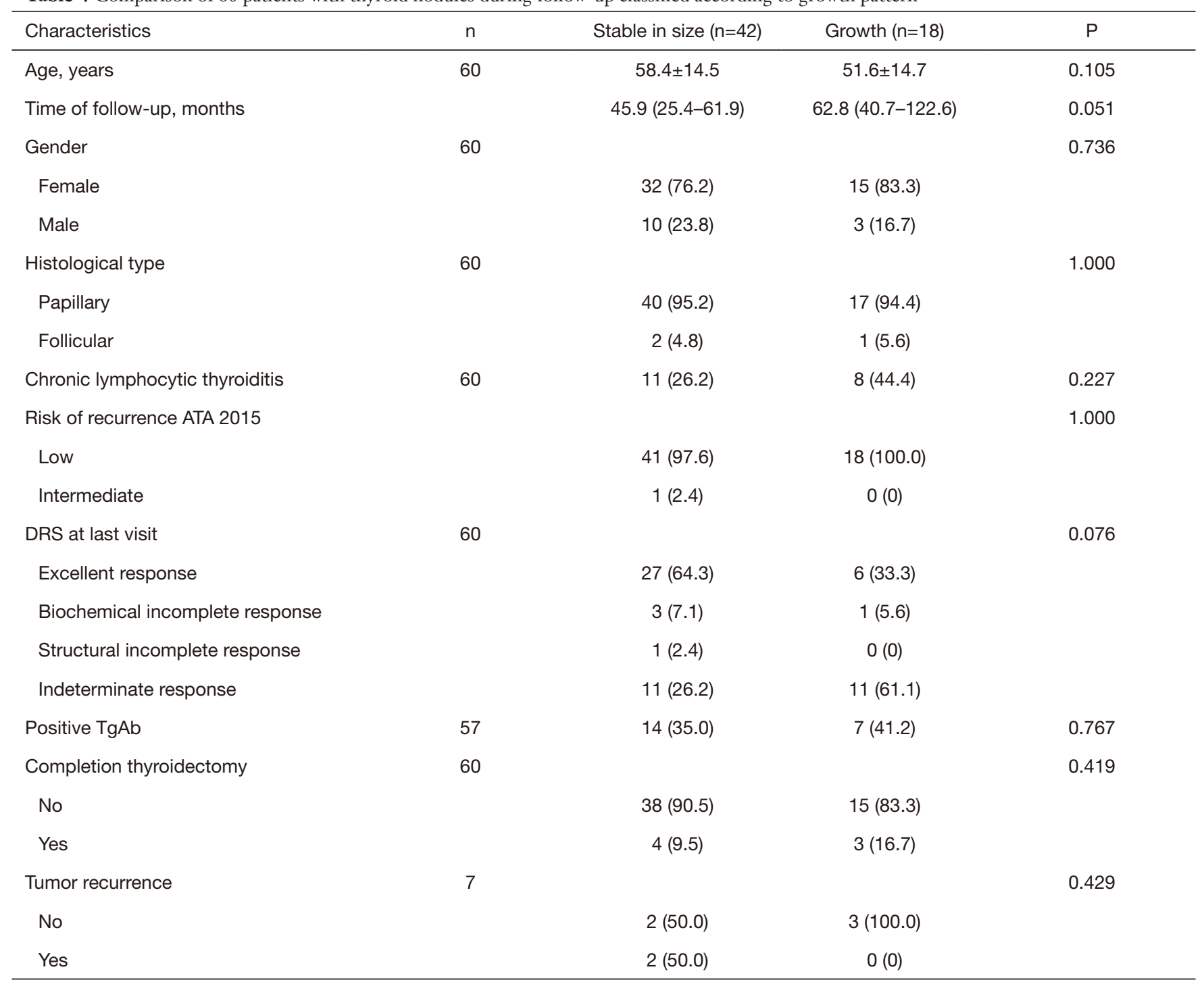

Data are the mean \pm SD or the median (interquartile range) for quantitative variables, and the number (percentage) for categorical variables. $n$, number of subjects; ATA, American Thyroid Association; TgAb, thyroglobulin antibodies; DRS, dynamic risk stratification.

after the hemithyroidectomy and even a higher percentage (74.4\%) have no evidence of disease at the end of followup. Furthermore, at this time, only $1.2 \%$ of patients showed evidence of structural disease.

Of the 42 uncured patients, 34 had indeterminate response, in most cases owing to the presence of $\mathrm{TgAb}$. It is known that, after thyroidectomy, most of patients with TgAb exhibit a decrease of the titer of these antibodies or become negative throughout the follow-up (23). However, patients with TL have remnant thyroid tissue and this is a limitation for decreasing TgAb levels. Six patients exhibited biochemical incomplete response and only one need surgery to complete thyroidectomy according to this criterion. It is also known that many of these patients spontaneously evolve to no evidence of disease, and only $31 \%$ develop structural disease (17). Finally, in the 2 patients with structural incomplete response, it was decided to complete the thyroidectomy with the expected finding of tumor recurrence.

According to ATA guidelines recommendations, most of the patients of this Spanish cohort were low risk, unifocal, and without lymph node involvement. There were no cases 


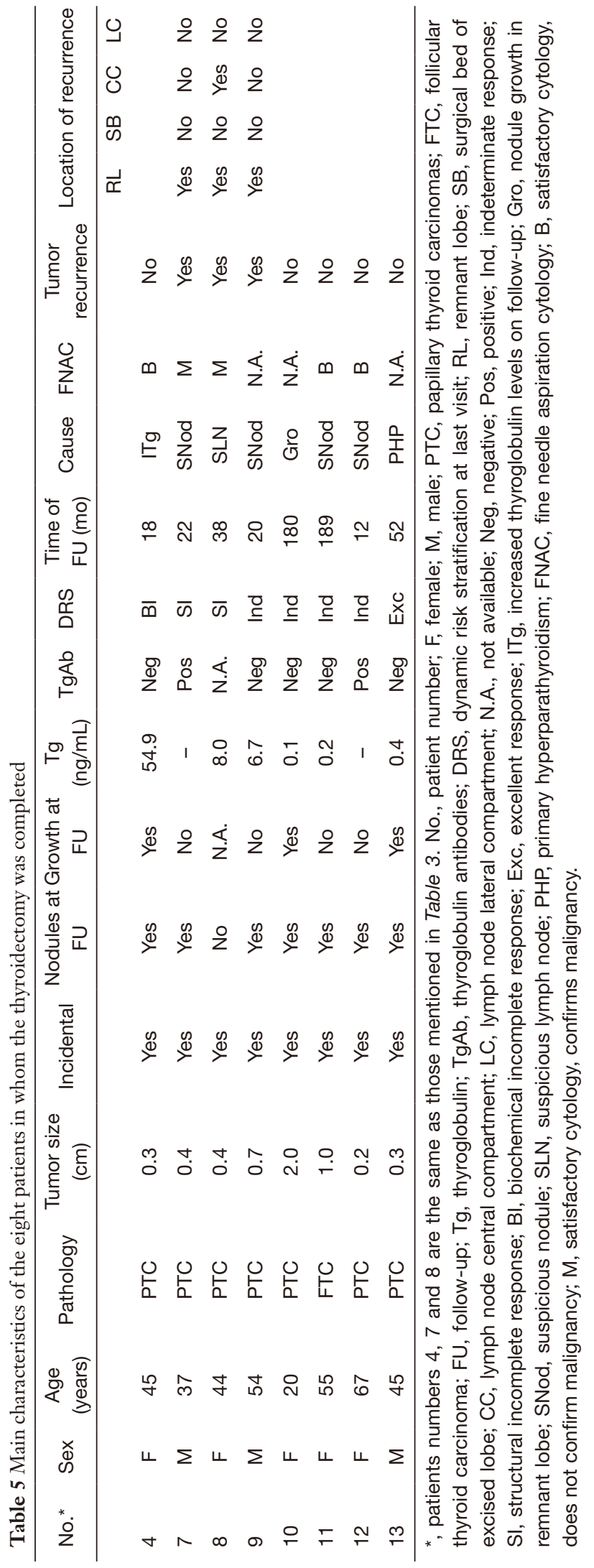

of extrathyroidal extension. One third had nodules in the contralateral lobe, although this is not a contraindication for TL. In fact, according to current recommendations, TL is sufficient for small, unifocal and intrathyroidal carcinomas in those patients with no history of craniocervical radiotherapy, family history of thyroid cancer, or clinically detectable lymph node metastases $(11,14)$. In the case of patients with minimally invasive FTC, 6 cases in our series, hemithyroidectomy is indicated in patients younger than 45 years of age, with tumor size $<4 \mathrm{~cm}$, and without vascular invasion, lymph node involvement or distant metastases (9).

We did not find significant differences between cured and non-cured patients at the end of follow-up, with the exception of a higher proportion of chronic lymphocytic thyroiditis in non-cured patients. This may be related to the fact that the presence of TgAb is a criterion for indeterminate response and lymphocytic thyroiditis is associated to the presence of these antibodies.

A remarkable fact is that only 8 of 164 (4.9\%) patients underwent second operation to complete thyroidectomy and, of these, in only $3(1.8 \%)$ the tumor recurrence was confirmed. This is in agreement with previous studies that have shown completion thyroidectomy rates as low as $10 \%$ and locorregional rates $<4 \%$ following LT (24).

Different authors have shown that the recurrence rate and overall survival of patients with low-risk DTC are not modified by the use of a more or less extensive surgical technique $(2,13,25)$. A study that used the NCDB (National Cancer Database) and included 61,775 patients with PTC of 1-4 cm, of which 54,926 underwent total thyroidectomy and 6,849 TL, showed a similar overall survival in both groups even after adjustment for demographic, clinical and pathologic factors (3). Other large epidemiological studies have yielded similar results $(2,26)$.

However, in a low-mortality disease such as DTC, it is essential to analyze, not only survival, but also the risk of recurrence. In a cohort of 2014 patients with papillary thyroid microcarcinoma (PTMC), after a propensity score matching, TL and total thyroidectomy were associated with similar long-term rates of death and recurrence (13). In another study in 889 patients with intrathyroid DTC, pT1-T2, no differences were found in overall survival or local or regional recurrences between those treated with TL $(41 \%)$ or total thyroidectomy (59\%) (10). The study by Kim et al. (27), in a group of 8,676 patients with PTMC, showed that total thyroidectomy reduced the risk of locoregional recurrence, but not that of recurrence outside the contralateral remnant lobe, except in multifocal 
cases. Nevertheless, other researchers did not find a higher rate of locoregional recurrences in multifocal PTCs treated with TL compared to those treated with total thyroidectomy $(8,28)$. In any case, given that recurrence in the remnant lobe can be adequately managed with the completion of the thyroidectomy, TL appears to be a reasonable option in patients with PTMC with or without multifocality. Furthermore, the rate of complications, including hypoparathyroidism and recurrent nerve palsy, has been shown to be higher in patients treated with total thyroidectomy compared to those undergoing TL $(4,8,29)$. Lastly, supporting the safety of a less aggressive surgical approach in low-risk patients, two recent meta-analyses $(30,31)$ have shown no statistically significant differences for recurrence rates, overall survival and disease-specific survival in low-risk DTC treated by total thyroidectomy or TL.

The ultrasound changes of the patients also deserve a comment. So far, ultrasound follow-up of patients with TL has been scarcely studied and there are no recommendations on its frequency of use. In our series, only 60 patients exhibited nodules during their followup and, of these, only 18 showed growth. Only 2 tumor recurrences were detected in the group of patients without nodular growth. Furthermore, we could not find any difference between patients with and without nodular growth. These findings are superimposable with those reported by Kim (32), who detected only 2 recurrences in a group of 137 patients with PTMC treated with TL. In another recent study (33), only $2 \%$ of 800 patients with PTMC treated with TL showed recurrence after 60 155 months of follow-up. The authors concluded that postsurgical ultrasound surveillance may be unnecessary due to the low recurrence rate in these patients.

$\mathrm{TL}$ is a mildly aggressive procedure that has the advantage of associating fewer perioperative complications. Preservation of the contralateral lobe and parathyroid glands is accompanied by a reduced need for replacement therapy with levothyroxine and calcium and vitamin $\mathrm{D}$ supplements (12). Hospitalization time and costs associated with TL are lower than those of a total thyroidectomy (34). Its main drawback is the risk of tumor recurrence in the remnant lobe $(11,12,35)$, which in our cohort reached $1,8 \%$, in line with that reported by recent studies $(27,29,32)$.

The strengths of our study lie in its multicenter nature and in that it provides, for the first time, an overview of the results of TL in patients with low-risk DTC in real clinical practice in our country. The limitations derive from a relatively small sample size and duration of followup. This may be probably due to the fact that TL has had little diffusion among Spanish surgical departments. Our study does not allow to compare the outcomes of patients undergoing TL with those treated by total thyroidectomy, since the latter were not included. To compare TL to total thyroidectomy, a larger sample size and a long-term follow up period should be needed. Most of the patients in this cohort had tumors $\leq 1 \mathrm{~cm}(93.3 \%)$ and many were incidental $(77.4 \%)$, therefore, our results cannot be extrapolated to populations with higher risk tumors. Besides, central lymph node dissection was performed in only 5 cases. The retrospective nature implies the loss of follow-up and the absence of data from some patients, as well as the use of different follow-up protocols in each hospital, and different criteria to decide whether or not to complete thyroidectomy. To ensure that results are reported as fully and accurately as possible we followed the STROBE guidelines (https://www. strobe-statement.org/index.php?id=strobe-home).

Obviously, not all patients with the presence of nodules or with nodular growth have undergone surgery, so we cannot assure that they are not carriers of persistence or recurrence of the disease. However, even if some of these patients have remnant disease, the clinical significance of this persistence should be minimal.

\section{Conclusions}

In summary, at the end of follow-up after a conservative management with no associated surgically related complications, $74.4 \%$ of our DTC patients achieved an excellent response and $98.8 \%$ showed no evidence of structural disease. Thyroidectomy was completed in $4.9 \%$ of the cohort, but only $1.8 \%$ showed tumor recurrence. These results, obtained in real clinical practice, support that the intensity of the treatment must be tailored to the risk of recurrence and mortality associated with each particular tumor and patient, in line with personalized medicine (36). This is especially important in low-risk patients since they are the most common in clinical practice and have traditionally been treated with more aggressive, and probably unnecessary, surgical procedures. In case of local recurrence in these patients, they can be safely managed by a second operation.

\section{Acknowledgments}

Funding: None. 


\section{Footnote}

Reporting Checklist: The authors have completed the STROBE reporting checklist. Available at http://dx.doi. org/10.21037/gs-20-712

Data Sharing Statement: Available at http://dx.doi. org/10.21037/gs-20-712

Peer Review File: Available at http://dx.doi.org/10.21037/gs20-712

Conflicts of Interest: All authors have completed the ICMJE uniform disclosure form (available at http://dx.doi. org/10.21037/gs-20-712). The authors have no conflicts of interest to declare.

Ethical Statement: The authors are accountable for all aspects of the work in ensuring that questions related to the accuracy or integrity of any part of the work are appropriately investigated and resolved. This study was conducted in accordance with the Declaration of Helsinki (as revised in 2013). The study was approved by the board of directors of the Thyroid Area of the Spanish Society of Endocrinology and Nutrition (TiroSEEN) and the ethics committee of the Hospital Universitario Ramón y Cajal (Madrid) (Acta 334, 27/11/17). Since this is a nonintervention and exclusively retrospective analysis of data from routine clinical practice, the informed consent of the patients has not been considered necessary.

Open Access Statement: This is an Open Access article distributed in accordance with the Creative Commons Attribution-NonCommercial-NoDerivs 4.0 International License (CC BY-NC-ND 4.0), which permits the noncommercial replication and distribution of the article with the strict proviso that no changes or edits are made and the original work is properly cited (including links to both the formal publication through the relevant DOI and the license). See: https://creativecommons.org/licenses/by-nc-nd/4.0/.

\section{References}

1. Haigh PI, Urbach DR, Rostein LE. Extent of thyroidectomy is not a major determinant of survival in low- or high-risk thyroid cancer. Ann Surg Oncol 2005;12:81-9.

2. Mendelsohn AH, Elashoff DA, Abemayor E, et al. Surgery for papillary thyroid carcinoma: is lobectomy enough? Arch Otolaryngol Head Neck Surg 2010;136:1055-61.

3. Adam MA, Pura J, Gu L, et al. Extent of surgery for papillary thyroid cancer is not associated with survival. An analysis of 61.775 patients. Ann Surg 2014;260:601-5; discussion 605-7.

4. Kim MJ, Lee MC, Lee GH, et al. Extent of surgery did not affect recurrence during 7-years follow-up in papillary thyroid cancer sized 1-4 cm: Preliminary results. Clin Endocrinol (Oxf) 2017;87:80-6.

5. McDougall IR, Camargo CA. Treatment of micropapillary carcinoma of the thyroid: where do we draw the line? Thyroid 2007;17:1093-6.

6. Hay ID, McConahey WM, Goellner JR. Managing patients with papillary thyroid carcinoma: insights gained from the Mayo Clinic's experience of treating 2.512 consecutive patients during 1940 through 2000. Trans Am Clin Climatol Assoc 2002;113:241-60.

7. Yu XM, Wan Y, Sippel RS, et al. Should all papillary thyroid microcarcinomas be aggressively treated? An analysis of 18.445 cases. Ann Surg 2011;254:653-60.

8. Jeon YW, Gwak HG, Lim ST, et al. Long-term prognosis of unilateral and multifocal papillary thyroid microcarcinoma after unilateral lobectomy versus total thyroidectomy. Ann Surg Oncol 2019;26:2952-8.

9. Dionigi G, Kraimps JL, Schmid KW, et al. Minimally invasive follicular thyroid cancer (MIFTC)-a consensus report of the European Society of Endocrine Surgeons (ESES). Langenbecks Arch Surg 2014;399:165-84.

10. Nixon IJ, Ganly I, Patel SG, et al. Thyroid lobectomy for treatment of well differentiated intrathyroid malignancy. Surgery 2012;151:571-9.

11. Haugen BR, Alexander EK, Bible KC, et al. 2015 American Thyroid Association Management Guidelines for Adult Patients with Thyroid Nodules and Differentiated Thyroid Cancer: The American Thyroid Association Guidelines Task Force on Thyroid Nodules and Differentiated Thyroid Cancer. Thyroid 2016;26:1-133.

12. Matsuzu K, Sugino K, Masudo K, et al. Thyroid lobectomy for papillary thyroid cancer: long-term followup study of 1,088 cases. World J Surg 2014;38:68-79.

13. Lee J, Park JH, Lee CR, et al. Long-term outcomes of total thyroidectomy versus thyroid lobectomy for papillary thyroid microcarcinoma: comparative analysis after propensity score matching. Thyroid 2013;23:1408-15.

14. Díez JJ, Oleaga A, Álvarez-Escolá C, et al. Guía clínica para el manejo de pacientes con carcinoma diferenciado de 
tiroides de bajo riesgo. Endocrinol Nutr 2015;62:e57-72.

15. Anda Apiñániz E, Zafon C, Ruiz Rey I, et al. The extent of surgery for low-risk $1-4 \mathrm{~cm}$ papillary thyroid carcinoma: a catch-22 situation. A retrospective analysis of 497 patients based on the 2015 ATA Guidelines recommendation 35 . Endocrine 2020;70:538-43.

16. Tuttle RM, Tala H, Shah J, et al. Estimating risk of recurrence in differentiated thyroid cancer after total thyroidectomy and radioactive iodine remnant ablation: using response to therapy variables to modify the initial risk estimates predicted by the new American Thyroid Association staging system. Thyroid 2010;20:1341-9.

17. Momesso DP, Vaisman F, Yang SP, et al. Dynamic risk stratification in patients with differentiated thyroid cancer treated without radioactive iodine. J Clin Endocrinol Metab 2016;101:2692-700.

18. Tuttle RM, Morris LF, Haugen BR, et al. ThyroidDifferentiated and anaplastic carcinoma. In: Amin MB, Edge SB, Greene FL, et al. editors. AJCC Cancer Staging Manual, Eighth ed. doi 10.1007/978-3-319-40618-3_73, 2017.

19. Momesso DP, Tuttle RM. Update on differentiated thyroid cancer staging. Endocrinol Metab Clin North Am 2014;43:401-21.

20. Campanella P, Ianni F, Rota CA, et al. Quantification of cancer risk of each clinical and ultrasonographic suspicious feature of thyroid nodules: a systematic review and metaanalysis. Eur J Endocrinol 2014;170:R203-11.

21. Brito JP, Gionfriddo MR, Al Nofal A, et al. The accuracy of thyroid nodule ultrasound to predict thyroid cancer: systematic review and meta-analysis. J Clin Endocrinol Metab 2014;99:1253-63.

22. Yeh MW, Bauer AJ, Bernet VA, et al. American Thyroid Association statement on preoperative imaging for thyroid cancer surgery. Thyroid 2015;25:3-14.

23. Spencer C, LoPresti J, Fatemi S. How sensitive (secondgeneration) thyroglobulin measurement is changing paradigms for monitoring patients with differentiated thyroid cancer, in the absence or presence of thyroglobulin autoantibodies. Curr Opin Endocrinol Diabetes Obes 2014;21:394-404.

24. Vargas-Pinto S, Romero Arenas MA. Lobectomy compared to total thyroidectomy for low-risk papillary thyroid cancer: a systematic review. J Surg Res 2019;242:244-51.

25. Ebina A, Sugitani I, Fujimoto Y, et al. Risk-adapted management of papillary thyroid carcinoma according to our own risk group classification system: is thyroid lobectomy the treatment of choice for low-risk patients? Surgery 2014;156:1579-88.

26. Adam MA, Pura J, Goffredo P, et al. Impact of extent of surgery on survival for papillary thyroid cancer patients younger than 45 years. J Clin Endocrinol Metab 2015;100:115-21.

27. Kim SK, Park I, Woo JW, et al. Total thyroidectomy versus lobectomy in conventional papillary thyroid microcarcinoma: analysis of 8.676 patients at a single institution. Surgery 2017;161:485-92.

28. Harries V, Wang LY, McGill M, et al. Should multifocality be an indication for completion thyroidectomy in papillary thyroid carcinoma? Surgery 2020;167:10-7.

29. Kwon H, Jeon MJ, Kim WG, et al. A comparison of lobectomy and total thyroidectomy in patients with papillary thyroid microcarcinoma: a retrospective individual risk factor-matched cohort study. Eur J Endocrinol 2017;176:371-8.

30. Bojoga A, Koot A, Bonenkamp J, et al. The impact of the extent of surgery on the long-term outcomes of patients with low-risk differentiated non-medullary thyroid cancer: a systematic meta-analysis. J Clin Med 2020;9:E2316.

31. van Gerwen M, Alsen M, Lee E, et al. Recurrencefree survival after total thyroidectomy and lobectomy in patients with papillary thyroid microcarcinoma. J Endocrinol Invest 2020. [Epub ahead of print].

32. Kim DW. Long-term follow-up ultrasonography after lobectomy in papillary thyroid microcarcinoma patients: A single-center study. Endocr Res 2016;41:213-7.

33. Baek HJ, Kim DW, Lee CY, et al. Analysis of postoperative ultrasonography surveillance after hemithyroidectomy in patients with papillary thyroid microcarcinoma: a multicenter study. Endocr Pract 2017;23:794-802.

34. Gibelli B, Dionisio R, Ansarin M. Role of hemithyroidectomy in differentiated thyroid cancer. Curr Opin Otolaryngol Head Neck Surg 2015;23:99-106.

35. Macedo FI, Mittal VK. Total thyroidectomy versus 
lobectomy as initial operation for small unilateral papillary thyroid carcinoma: a meta-analysis. Surg Oncol 2015;24:117-22.

Cite this article as: Díez JJ, Alcázar V, Iglesias P, Romero-Lluch A, Sastre J, Corral BP, Zafón C, Galofré JC, Pamplona MJ. Thyroid lobectomy in patients with differentiated thyroid cancer: an analysis of the clinical outcomes in a nationwide multicenter study. Gland Surg 2021;10(2):678-689. doi: 10.21037/gs-20-712
36. Zafón C, Díez JJ, Galofré JC, et al. Nodular thyroid disease and thyroid cancer in the era of precision Medicine. Eur Thyroid J 2017;6:65-74. 


\section{Supplementary}

Table S1 Results of the dynamic risk stratification at 12 months and at the end of follow-up in patients with available data

\begin{tabular}{|c|c|c|c|c|}
\hline Type of response & \multicolumn{2}{|c|}{12 months $(n=148)$} & \multicolumn{2}{|c|}{ Last visit $(n=164)$} \\
\hline Excellent & 106 & 71.6 & 122 & 74.4 \\
\hline Indeterminate & 36 & 24.3 & 34 & 20.7 \\
\hline Biochemical incomplete & 5 & 3.4 & 6 & 3.7 \\
\hline
\end{tabular}

n, number of subjects.

Table S2 Comparison of 148 patients at the end of the first year of follow-up classified according to absence (excellent response) or presence of disease (any other type of response)

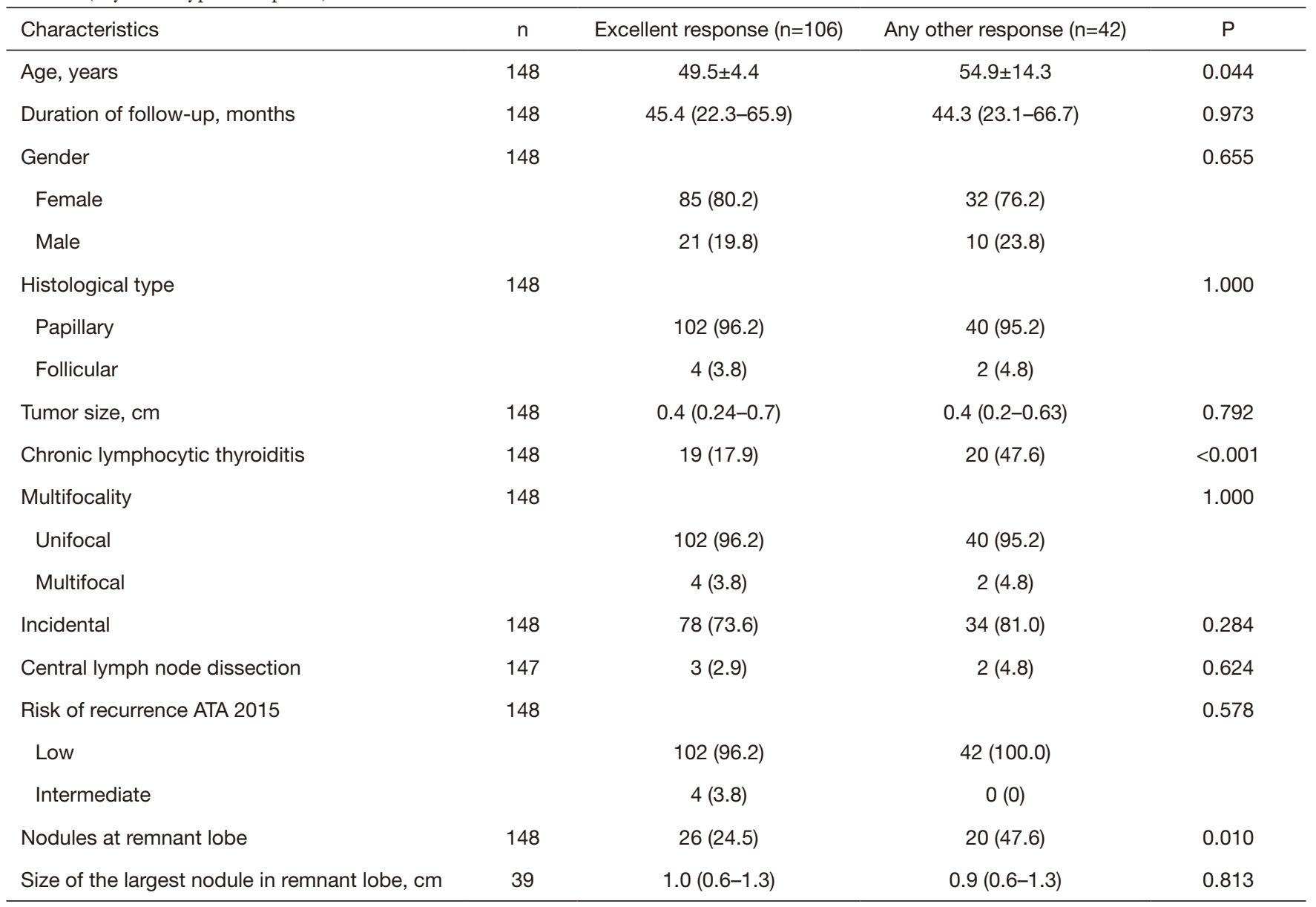

Data are the mean \pm SD or the median (interquartile range) for quantitative variables, and the number (percentage) for categorical variables. $n$, number of subjects; ATA, American Thyroid Association. 


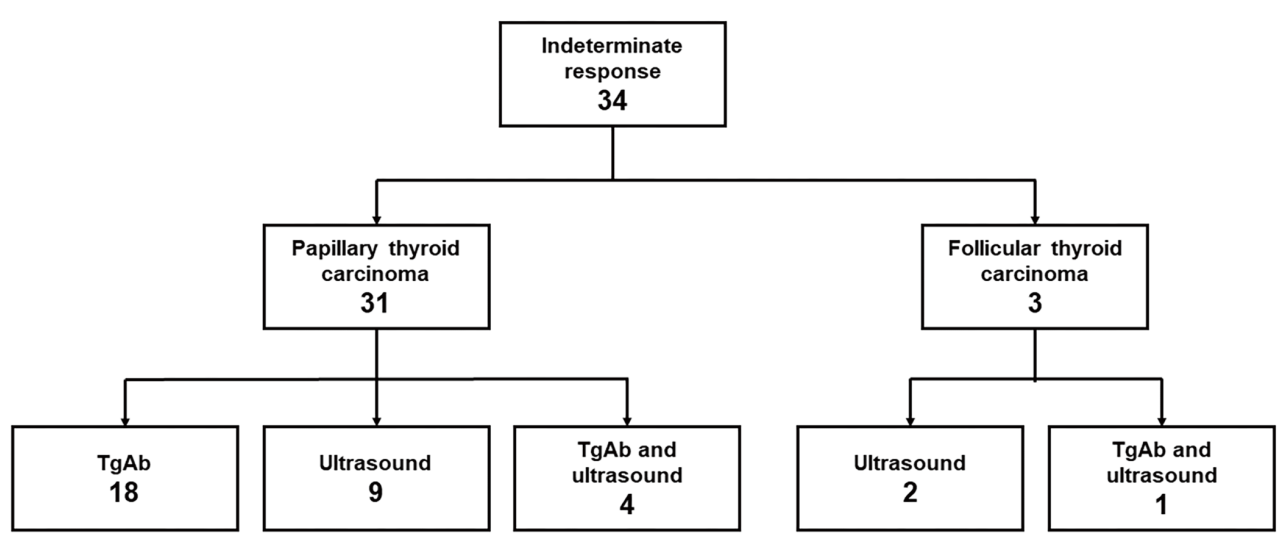

Figure S1 Diagram indicating the criteria for indeterminate response at the end of follow-up in patients with papillary and follicular thyroid carcinoma. TgAb, thyroglobulin antibodies.

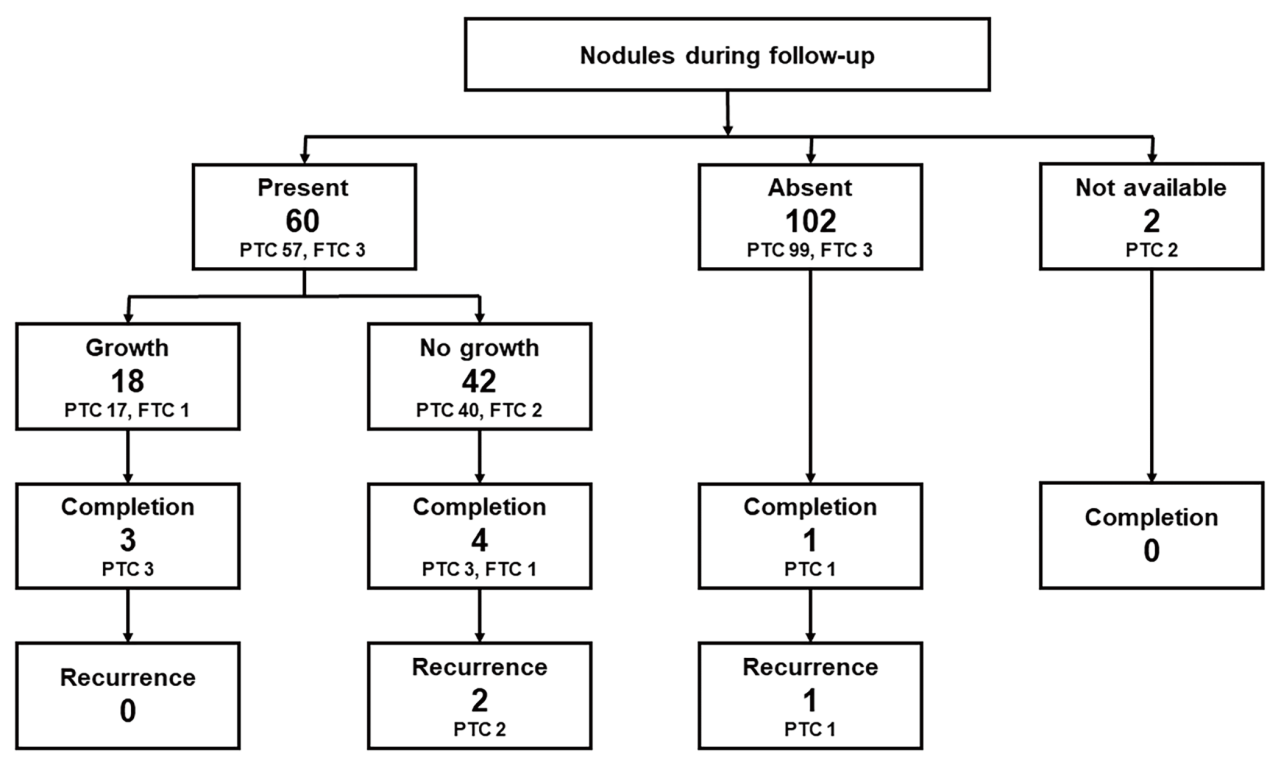

Figure S2 Diagram indicating the evolution of patients classified according to the presence or absence of nodules during follow-up. The reason for completing the thyroidectomy in the patient with no nodules during follow-up was the presence of suspicious cervical lymph nodes. PTC, papillary thyroid carcinoma; FTC, follicular thyroid carcinoma. 